Published in final edited form as:

Virus Res. 2006 December ; 122(1-2): 144-153.

\title{
Members of adenovirus species B utilize CD80 and CD86 as cellular attachment receptors
}

\author{
Joshua J. Short ${ }^{a}$, Chenthamarakshan Vasu $^{\mathrm{b}}$, Mark J. Holterman ${ }^{\mathrm{b}}$, David T. Curiela ${ }^{\mathrm{a}}$, and \\ Alexander Pereboeva, ${ }^{*}$ \\ a Division of Human Gene Therapy, Departments of Medicine, Obstetrics and Gynecology, Pathology, and \\ Surgery, and the Gene Therapy Center, University of Alabama at Birmingham, Birmingham, AL 35294-2172, \\ USA \\ b Departments of Surgery, and Microbiology and Immunology, University of Illinois at Chicago, Chicago, \\ IL 60612, USA
}

\section{Abstract}

\begin{abstract}
Alternate serotypes of adenovirus (Ad), including Ads of species B, are being explored to circumvent the disadvantages of Ad serotype 5 gene delivery vectors. Whereas the majority of human Ads utilize the Coxsackievirus and adenovirus receptor (CAR), none of the Ad species B use CAR. Ad species $\mathrm{B}$ is further divided into two subspecies, $\mathrm{B} 1$ and $\mathrm{B} 2$, and utilizes at least two classes of receptors: common Ad species B receptors and B2 specific receptors. CD46 has been implicated as a B2-specific receptor. Ad serotype 3 (Ad3), a member of B1, utilizes CD80 and CD86 as cellular attachment receptors. The receptor-interacting Ad fiber-knob domain is highly homologous among species $\mathrm{B}$ Ads. We hypothesized that other members of Ad species B may utilize CD80 and CD86 as cellular attachment receptors. All tested species B members showed specific binding to cells expressing CD80 and CD86, and the Ad fiber-knob domain from both B1 and B2 Ad efficiently blocked CD80- and CD86-mediated infection of Ad3 vectors. Members of both B1 and B2 demonstrated CD80- and CD86-specific infection of $\mathrm{CHO}$ cells expressing CD80 and CD86. Therefore, all of the members of Ad species B utilize CD80 and CD86 for infection of cells.
\end{abstract}

\section{Keywords}

Adenovirus species B; Receptor; CD80; CD86

\section{Introduction}

\begin{abstract}
Adenoviruses are non-enveloped, double-stranded DNA viruses with icosahedral symmetry. To date, there are 51 different human Ad serotypes, which are classified into six different species (A-F) based on their biological and genetic properties (Russell, 2000). Most Ad serotypes infect cells via a two-step process: (i) Ad binding to a primary cellular attachment receptor mediated by the distal knob domain of fiber protein extending from the Ad capsid vertices (Louis et al., 1994) and by (ii) the ensuing interaction of $\alpha_{V}$ integrins on the cell surface and the Ad penton base Arg-Gly-Asp (RGD) motif (Wickham et al., 1993). The initial binding of Ad to their primary receptor is thought to be one of the key determinants of virus tropism. Most human Ads cause disease in the respiratory tract, kidneys, eyes, intestine, or lymphoid tissue. The vast majority of the serotypes, including Ad serotypes 2 (Ad2) and 5 (Ad5), utilize CAR as their primary cellular attachment receptor (Bergelson et al., 1997; Roelvink et al.,
\end{abstract}

* Correspondance to: BMR-2, 901, 19th Street, Room 406, Birmingham AL 35294-2172, USA. Tel.: +1 205975 8734; fax: +1 205975 8565., E-mail address: pereboev@uab.edu (A. Pereboev).. 
1998; Tomko et al., 2000). Ad2 and Ad5 are the most thoroughly studied serotypes and the most commonly used Ad serotypes in gene therapy applications.

It is noteworthy that Ads have been exploited as gene transfer vectors. It is unfortunate that gene therapy protocols employing Ad5-based vectors have resulted in limited efficacies (Rancourt et al., 1998; Sterman et al., 1998). This may be in part due to deficient/low CAR expression or inaccessibility of CAR on the cell surface in the tissues of interest. CAR has been identified as a tumor suppressor protein (Kim et al., 2003; Okegawa et al., 2000) and a member of the intracellular junction (Cohen et al., 2001; Walters et al., 2002). CAR is often downregulated in neoplastic conversion; when expressed, its location basal to the tight junction may limit its accessibility. Efforts to circumvent these deficiencies have prompted the investigation of other non-CAR-binding Ad serotypes. One method of accomplishing such an investigation is by the construction of pseudotyped Ad vectors (Krasnykh et al., 1996). Pseudotyped Ad vectors replace the fiber-knob domain of the Ad5 vector with the fiber-knob domain from a non-CAR binding serotype, thereby achieving serotype-specific infection of cells. Vectors pseudotyped with the fiber-knob domain from serotypes of Ad species B, such as Ad serotype 3 (Ad3), efficiently transduce a variety of malignant cell types (Davidoff et al., 1999; Kanerva et al., 2002; Von Seggern et al., 2000) as well as many other target cells of interest (Stevenson et al., 1997). Until recently, further development and characterization of Ad species B-based vectors had been hindered by the failure to identify their primary cellular attachment receptors.

Species B is subdivided into two subspecies: B1, consisting of serotypes 3, 7, 16, 21, and 50; and B2, consisting of serotypes $11,14,34$, and 35 . There are at least two classes of species B Ad receptors: common species B Ad receptors utilized by all species B Ad and B2-specific receptors (Segerman et al., 2003a); however, none of the species B Ads utilize CAR as their primary cellular attachment receptor (Roelvink et al., 1998). Viruses from subspecies B2 can fully inhibit the binding of subspecies B1 viruses; however, viruses from subspecies B1 can only partially inhibit the binding of subspecies B2 viruses (Segerman et al., 2003a). CD46 was identified as a cellular attachment receptor for Ad serotypes 11 (Ad11) and 35 (Ad35), and both are members of the subspecies B2 (Gaggar et al., 2003; Segerman et al., 2003b). Additionally, all of subspecies B2 Ad as well as most of the subspecies B1 Ad are capable of binding to CD46 expressed on the cell surface. However, only binding of the virions to CD46 was demonstrated; transduction was not (Gaggar et al., 2003). Ad serotype 7 (Ad7), a member of subspecies B1, is capable of binding to CD46 but it cannot infect- $\mathrm{CHO}$ cells expressing CD46, leading to the hypothesis that CD46 serves as a subspecies B2-specific receptor (Segerman et al., 2003b). However, contrary to this hypothesis, Ad3 has recently been shown capable of using CD46 as a cellular attachment receptor, thereby complicating the picture of Ad species B receptors (Sirena et al., 2004). Furthermore, we have recently demonstrated that Ad3 is also capable of utilizing CD80 (B7.1) and CD86 (B7.2) as cellular attachment receptors (Short et al., 2004).

CD80 and CD86 are cell surface markers expressed on human dendritic cells and mature Blymphocytes (Caux et al., 1994; Engel et al., 1994; Freeman et al., 1989; Lanier et al., 1995), and play a critical role in stimulating T-lymphocyte responses by their interaction with the ligands CD28 and CTLA-4 (Azuma et al., 1993; Caux et al., 1994; Lanier et al., 1995; Vasu et al., 2003). Both CD80 and CD86 are up-regulated during the process of dendritic cell maturation and are over-expressed in a variety of lymphocytic neoplasms and other neoplastic contexts (Koyama et al., 1998; Maeda et al., 2000; Mutti et al., 1998). Whereas CD80 and CD86 serve as a primary means of cellular entry for Ad3 on human dendritic cells, there may be additional cellular receptors for Ad 3 capable of mediating the infection of HeLa cells (Short et al., 2004). 
To better understand the cellular receptors utilized by Ad species B, we investigated other serotypes of Ad species B for their ability to utilize CD80 and CD86. The Ad fiber-knob domain is highly homologous among species B Ad. On this basis, we hypothesized that other members of Ad species B may utilize CD80 and CD86 as cellular attachment receptors.

\section{Materials and methods}

\subsection{Cell culture}

Chinese hamster ovary (CHO) cell line and HeLa cell line (both from ATCC, Manassas, VA) were grown in DMEM/F12 50:50 with 10\% FBS and antibiotics as recommended by the provider. Generation of CHO-CD80 and CHO-CD86 cell lines and characterization of ligand expression levels were reported earlier (Short et al., 2004; Vasu et al., 2003). The cells were grown in the same media in the presence of geneticin at $400 \mu \mathrm{g} / \mathrm{ml}$. All cells were cultured in a humidified incubator at $37{ }^{\circ} \mathrm{C}$ with $5 \% \mathrm{CO}_{2}$.

\subsection{Wild-type and recombinant Ad}

Wild-type Ad serotypes 3, 5, 7, 11, 14, 16, 21, 34, 35, and 50 (ATCC) were amplified and titrated spectrophotometrically (Maizel et al., 1968). Two replication-incompetent Ad5-based vectors containing a firefly luciferase transgene cassette in place of the deleted E1 region were used. Ad5luc1 contains the full Ad5 capsid and fiber proteins whereas Ad5/3luc1 contains the Ad5 capsid with chimeric fiber proteins in which the tail and shaft domains are from Ad5 and the knob domain is from Ad3. Both vectors were generated in our laboratory and described previously (Kanerva et al., 2002).

\subsection{Recombinant $\mathrm{Ad} 3$ and $\mathrm{Ad} 5$ fiber-knob domain and $\mathrm{Ad} 7$ and $\mathrm{Ad} 11$ fiber protein production}

The knob domains of Ad5 and Ad3 fibers (Krasnykh et al., 1996) and the fibers of Ad7 and Ad11 were produced in $E$. coli with N-terminal tags of six consecutive histidine residues (6HIS), using the pQE30 expression vector (Qiagen, Valencia, CA). Ad7 and Ad11 wild-type genomes were used as templates for PCR to amplify the fiber genes of the respective serotype. Primers for these reactions were Ad7F11F.F (5'-TAC CCC GGG AAT GAC CAA GAG AGT CCG G-3'), Ad7F.R (5'-ATT AAG CTT TCA GTC GTC TTC TCT AAT G-3'), Ad11F.R (5'ATT AAG CTT TCA GTC GTC TTC TCT GAT G-3'). The same forward primer was used in both reactions but with each reverse primer a DNA sequence was amplified coding for the fiber polypeptide of the respective serotype. Both PCR products were then digested with Sma I and Hind III and cloned into Sma I-Hind III-digested pQE30, resulting in plasmids pQE.Ad7F and pQE.Ad11F further used to transform E. coli. The soluble forms of the Ad3 and Ad5 fiber-knob domain and Ad7 and Ad11 fiber proteins were isolated from the induced E. coli cultures using the BugBuster Protein Extraction Reagent (Novagen, Madison, WI). The proteins were further purified using the Talon metal affinity resin (Clontech, Palo Alto, CA) as recommended by the manufacturer. To further increase the protein yield, the expressed Ad3 fiber-knob domain protein was purified from inclusion bodies followed by refolding using the BugBuster Protein Extraction Reagent inclusion body protocol as recommended by the manufacturer. The concentration of the purified proteins was determined by the Bio-Rad protein assay (Bio-Rad, Hercules, CA). The ability of each Ad fiber-knob domain or fiber to form a homotrimer was verified by Western blot of unboiled versus boiled samples using mouse monoclonal anti-polyhistidine clone HIS-1 antibody (Sigma, St. Louis, MO). Ad fiber-knob domain and fiber function was confirmed by its ability to inhibit Ad infection of the same serotype but not affect infection of the other species. 


\subsection{Flow cytometry}

In order to assess binding of recombinantly produced Ad fiber to CD80 and CD86 expressed on the cell surface, CHO-CD80 and CHO-CD86 cells were grown to approximately $85 \%$ confluency, harvested with Versene (UAB Media Preparation Facility), and portions of $10^{5}$ cells incubated with Ad7 fiber, Ad11 fiber, or without Ad fiber in growth media for $1 \mathrm{~h}$ at $4^{\circ}$ C. Cells were then washed 3 times in cold $\left(4^{\circ} \mathrm{C}\right) \mathrm{PBS}$ with $\mathrm{Ca}^{++}$and $\mathrm{Mg}^{++}(\mathrm{PBSCM})$. Cells were next incubated with mAb HIS-1 in PBSCM plus $1 \%$ BSA for $1 \mathrm{~h}$ at $4{ }^{\circ} \mathrm{C}$. Cells were washed 3 times in cold PBSCM and incubated with goat anti-mouse $\mathrm{IgG}$ antibodies conjugated to a green fluorophore, Alexa 488 (Molecular Probes, Eugene, OR), in PBSCM plus 1\% BSA for $1 \mathrm{~h}$ at $4{ }^{\circ} \mathrm{C}$. All of the cells were washed 3 final times in cold PBSCM and analyzed by flow cytometry by the Center for AIDS Research FACS Core Facility at UAB. For detection of Ad virion binding, cells were harvested with Versene and portions of $10^{5}$ cells incubated without virus or with 100 viral particles (vp)/cell of Ad3, Ad5, Ad7, Ad11, Ad14, Ad16, Ad21, Ad34, $\mathrm{Ad} 35$, or Ad50 in growth media for $1.5 \mathrm{~h}$ at $4{ }^{\circ} \mathrm{C}$. Cells were washed 3 times in cold PBSCM and incubated with anti-Ad hexon rabbit polyclonal antibody (UAB) in PBSCM plus 1\% BSA for $1 \mathrm{~h}$ at $4{ }^{\circ} \mathrm{C}$. Cells were washed 3 times in cold PBSCM and incubated with anti-rabbit IgG antibodies conjugated to a fluorophore, Alexa 488, in PBSCM plus $1 \%$ BSA for $1 \mathrm{~h}$ at $4{ }^{\circ} \mathrm{C}$. All of the cells were washed 3 final times in cold PBSCM and analyzed by flow cytometry. The labeled cells were gated and scored as positive or negative and the percent of positive cells were plotted for each virus.

\subsection{ELISA}

Indicated amounts of recombinant Ad3 or Ad5 fiber-knob or Ad7 or Ad11 fiber were adsorbed on a 96 well plate for $30 \mathrm{~min}$ at $37^{\circ} \mathrm{C}$. Wells were then washed with Tris buffered saline with Tween-20 (TBST) and blocked with $0.5 \%$ casein in TBST for $30 \mathrm{~min}$ at $37^{\circ} \mathrm{C}$. After washing with TBST, $0.2 \mu \mathrm{g} /$ well of recombinant extracellular domains of CD80 or CD86 fused with Fc region of human IgG1 (sCD80, sCD86) (R\&D Systems Inc., Minneapolis, MN) in PBSCM plus $1 \%$ BSA was added to each well for $30 \mathrm{~min}$ at $37^{\circ} \mathrm{C}$. Wells were then washed 3 times with TBST and incubated with anti-human IgG antibodies conjugated to alkaline phosphatase (DAKO Corporation) in PBSCM plus $1 \%$ BSA for $30 \mathrm{~min}$ at $37^{\circ} \mathrm{C}$. Wells were washed 3 times with TBST and developed with $p$-nitrophenyl phosphate (Sigma-Aldrich). Alkaline phosphatase activity was assayed by measuring the optical density at $405 \mathrm{~nm}$.

\subsection{Gene transfer analysis}

Cells were seeded in 48 well plates and allowed to grow until $85 \%$ confluent. The cells were then incubated with Ad vectors (100 vp/cell) or no virus in DMEM:F12 50:50 with 2\% FBS for $1.5 \mathrm{~h}$ at $37^{\circ} \mathrm{C}$. For blocking experiments, the cells were pre-incubated with recombinant Ad7 or Ad1 1 fiber $(0.1,0.05,0.025 \mathrm{mg} / \mathrm{ml})$. For all blocking trials, incubation occurred for 10 min at $37^{\circ} \mathrm{C}$ before virus was incubated with the cells for $1.5 \mathrm{~h}$ at $37^{\circ} \mathrm{C}$. Virus was then removed and the cells were rinsed with PBS and grown in growth media. After $36 \mathrm{~h}$ the cells were lysed and the luciferase assay was performed with the Luciferase Assay System (Promega, Madison, WI). The protein concentration in the cell lysates was determined with DC Bio-Rad protein assay to allow normalization of the gene expression data to the number of cells. Background luciferase activities were subtracted from the readings. All of the samples were done in triplicate and the mean average and standard deviation were calculated.

\subsection{RT-PCR analysis}

Cells were seeded in 24 well plates and grown to $85 \%$ confluency. Cells were incubated without virus or with wild-type Ad3, Ad7, Ad11, Ad14, Ad21, Ad35, and Ad50 at 10 vp/cell for $1.5 \mathrm{~h}$ at $37^{\circ} \mathrm{C}$. The cells were rinsed with PBS and grown in growth media for $6 \mathrm{~h}$. The cells were washed and total cellular RNA was extracted using RNeasy RNA Isolation Kit (Qiagen) per 
the manufacturer's instructions. Nucleotide sequence identity existing within the E1A genes of the B1 and B2 species allowed only two sets of primers for detection of E1A: a pair B1 E1A and a pair B2 E1A (below). Primers were also designed for the detection of the mRNA of CD80, CD86, and actin. The actin primers used were suitable for recognizing human and hamster actin. RT-PCR was performed using the following primers: B1 E1A (5'-TGA GAC ACC TGC GCT TCC TG-3', 5'-TCA TTG CCT TGG CAG TTT CCG-3'), B2 E1A (5'-ATG AGA GAT TTG CGA TTT CTG-3', 5'-TTA TTG TCT TGG ACG TTT CCG TG-3'), CD80 (5'-ATG GGC CAC ACA CGG AGG CAG-3', 5'-TTA TAC AGG GCG TAC ACT TTC-3'), CD86 (5'-ATG GAT CCC CAG TGC ACT ATG-3', 5'-TTA AAA ACA TGT ATC ACT TTT G-3'), Actin (5'-ATG GAT GAT GAT ATC GCC G-3', 5'-CTA GAA GCA TTT GCG GTG G-3') using One-Step RT-PCR kit following the protocol of the manufacturer (Qiagen).

\subsection{Immunofluorescence imaging}

Cells were seeded on sterilized $18 \mathrm{~mm} \times 18 \mathrm{~mm}$ cover slips and allowed to grow as described above until $85 \%$ confluent. The cells were then incubated without virus or infected with wildtype $\mathrm{Ad} 3, \mathrm{Ad} 5, \mathrm{Ad} 7, \mathrm{Ad} 11$, and $\mathrm{Ad} 35$ at $10 \mathrm{vp} / \mathrm{cell}$ for $1.5 \mathrm{~h}$ at $37^{\circ} \mathrm{C}$. The cells were rinsed with PBS and grown in growth media for $36 \mathrm{~h}$. The cover slips were rinsed with cold $\left(4^{\circ} \mathrm{C}\right)$ PBS, fixed with absolute methanol at $-20^{\circ} \mathrm{C}$ for $7 \mathrm{~min}$, rinsed with cold PBS, and post-fixed with $3 \%$ formaldehyde at room temperature (RT) for $15 \mathrm{~min}$. Following fixation the cells were rinsed and washed with four changes of PBS and blocked in blocking buffer (PBS with 1\% BSA) for $15 \mathrm{~min}$ at RT. The cells were then incubated with anti-Ad hexon rabbit polyclonal antibodies in blocking buffer for $1 \mathrm{~h}$ at RT. The cells were then rinsed and washed with four changes of PBS and blocked in blocking buffer for $15 \mathrm{~min}$ at RT. The cells were then incubated with goat anti-rabbit IgG conjugated to Alexa 488 for $1 \mathrm{~h}$ in blocking buffer at RT. The coverslips were finished by washing through four changes of PBS and then incubating in Hoechst 33342 (Molecular Probes) for 4 min. The cells were washed in PBS overnight at $4{ }^{\circ}$ C. The cover slips were mounted to glass slides in $0.1 \%$ p-phenylene diamine (PPD) (SigmaAldrich) in 1:9 PBS:glycerol, sealed with fingernail polish, and stored at $-20{ }^{\circ} \mathrm{C}$. Images were obtained through the high resolution imaging facility at UAB. To better view the staining on the printed images the green staining color was replaced with the color white.

\section{Results}

\subsection{Recombinant Ad fiber proteins from Ad subspecies B1 and B2 bind to cells expressing CD80 and CD86}

To assess the ability of Ad subspecies B1 (Ad7) and B2 (Ad11) to bind to CD80 and CD86, we incubated recombinant Ad fiber proteins with $\mathrm{CHO}-\mathrm{CD} 80$ and CHO-CD86 cells, followed by detection via flow cytometry (Fig. 1). Neither Ad fiber bound to the parental $\mathrm{CHO}$ cells, which do not express CD80 or CD86. However, expression of human CD80 or CD86 conferred the ability of both Ad fibers to bind to the CHO-CD80 and CHO-CD86 cells. The Ad11 fiber showed a slightly higher fluorescent labeling than the Ad7 fiber did on both cell lines. Both Ad fibers were also able to bind to HeLa cells, which express both CD80 and CD86 (Short et al., 2004, this study). However, on these cells the possibility of additional receptors, such as CD46 or other unidentified proteins could be responsible for the high level of binding.

\subsection{Ad fiber proteins from Ad subspecies B1 and B2 interact specifically with CD80 and CD86}

To confirm the ability of Ad fiber proteins from Ad subspecies B1 and B2 to interact with CD80 and CD86, we performed an ELISA to assay the interaction of the knob domain of Ad3, Ad7, Ad11, and the species C Ad5 fiber proteins with CD80 (Fig. 2A) and CD86 (Fig. 2B). The fiber proteins of all three Ad species B members, Ad3, Ad7, and Ad11, showed similar levels of specific binding to both CD80 and CD86, whereas Ad5 showed no binding with CD80 or CD86. 


\subsection{Recombinant Ad fiber proteins from Ad subspecies B1 and B2 specifically block CD80- and CD86-mediated gene transfer of Ad5/3 pseudotyped vectors}

Ad3 has previously been shown capable of infecting cells via CD80 and CD86 (Short et al., 2004). To further demonstrate the ability of Ad subspecies B1 and B2 members to interact with CD80 and CD86, we assayed the Ad fiber proteins from Ad7 and Ad11 for their ability to block CD80- and CD86-dependent transduction of an Ad5 vector pseudotyped with the Ad3 fiber-knob domain (Ad5/3luc1) (Fig. 3A and C). Both Ad7 and Ad11 fiber proteins were able to block Ad5/3luc1 transduction on the CHO-CD80 and CHO-CD86 cells; however, neither protein had any effect on transduction of the same cells by an Ad5 vector (Fig. 3B and D). The level of inhibition of the Ad5/3luc1 vector ranged from 80 to $90 \%$ on the CHO-CD80 and CHO-CD86 cells for each Ad fiber protein at a concentration of $100 \mu \mathrm{g} / \mathrm{ml}$. Additionally, Ad5/3luc1 gene transfer to HeLa cells was blocked by $95 \%$ by both Ad7 and Ad11 fiber proteins at a concentration of $100 \mu \mathrm{g} / \mathrm{ml}$.

\subsection{All members of Ad species B exhibit binding to cells expressing CD80 and CD86}

To further confirm the ability of whole virions to specifically bind to CD80 and CD86 on the cell surface, Ad species B were assayed by flow cytometry for their ability to bind to $\mathrm{CHO}$ cells expressing CD80 and CD86 (Fig. 4). HeLa cells were used as a positive control to confirm the ability of all viruses to successfully bind and be detected. This control also served as verification that the anti-Ad hexon polyclonal antibodies recognized all of the Ad hexon serotype variants. All members of the Ad species B were able to bind to the CD86-expressing cells at an increased level when compared to the parental CHO cells. In the case of CD80, all members were able to bind to the $\mathrm{CD} 80$-expressing cells to a significantly higher degree than the $\mathrm{CHO}$ counterpart cell lines but exhibited a lower level of binding when compared with the CHO-CD86 cells. There was no significant increase in binding of the Ad5 virus to any of the $\mathrm{CHO}$ cell derivatives versus the $\mathrm{CHO}$ parental cell line. The variation in the ability of the viruses to bind to the cells may be a result of varying affinities for CD80 and CD86 or of the ability of the anti-Ad hexon antibody to detect the Ad serotype.

\subsection{Expression of CD80 and CD86 on the cell surface enhances infection by members of $A d$ species $B$}

The expression of CD80 and CD86 by the HeLa, $\mathrm{CHO}$, and CHO-derivative cells was evaluated by RT-PCR (Fig. 5). PCR products of the expected size for CD80 (867 bp) and CD86 (990 bp) were detected with CD80- or CD86-specific primers, respectively, in the HeLa cells and the respective CHO-derivative cells, but not in the $\mathrm{CHO}$ or alternate $\mathrm{CHO}$-derivative cells (Fig. 5A and B). To assess the ability of members of Ad species B to infect cells via CD80 and CD86, CHO cells expressing CD80 or CD86 were infected with wild-type members of Ad subspecies B1 and B2. E1A RNA transcripts were detected by RT-PCR at $6 \mathrm{~h}$ post-infection for members of Ad subspecies B1 and B2, including serotypes 3, 7, 11, 14, 35, and 50, in the HeLa (Fig. 5C), CHO (Fig. 5D), CHO-CD80 (Fig. 5E), and CHO-CD86 (Fig. 5F) cells. Ad5 was capable of infecting the HeLa cells, but no E1A transcripts were detected in any of the CHO cells. Actin RNA transcripts were also detected to ensure an appropriate level of RNA was used in each sample. All of the cells were infected with equal quantities of virus particles; however, we did not quantify gene expression in these experiments due to the level of sensitivity required. Qualitatively, there was a clear increase in E1 A gene expression in $\mathrm{CHO}$ cells expressing CD80 and CD86 when compared with the parental CHO cells.

\subsection{Hexon accumulation of Ad species $B$ members in cells expressing CD80 and CD86}

To further demonstrate that CD80 and CD86 expression on CHO cells enhances infection by members of Ad species B, we used immunofluorescent staining after infection to investigate the accumulation of the Ad capsid protein hexon. Parental CHO cells without expression of 
CD80 or CD86, HeLa cells, and CHO cells expressing CD80 or CD86 were infected with wildtype virus of two members of Ad subspecies B1 (Ad3, Ad7) and two members from Ad subspecies B2 (Ad11, Ad35), as well as Ad5. After $36 \mathrm{~h}$, the cells were analyzed by immunofluorescence for the presence of the hexon protein (Fig. 6). Productive viral replication is not believed to occur in the non-human $\mathrm{CHO}$ cells; however, the accumulation of the $\mathrm{Ad}$ hexon protein was assayed, not productive viral particles. None of the $\mathrm{CHO}$ cells infected showed significant levels of hexon accumulation. There were few cells with minimal accumulation, presumably caused by non-specific background infection due to interactions between the RGD motif on the Ad capsid and cellular integrins. All of the viruses were able to infect the HeLa cells which resulted in an accumulation of hexon. After infection with the Ad5 virus, both the $\mathrm{CHO}-\mathrm{CD} 80$ and $\mathrm{CHO}-\mathrm{CD} 86$ cells showed a pattern of hexon accumulation similar to the pattern seen in the parental $\mathrm{CHO}$ cells with expression much lower than in the HeLa cells. In contrast, $\mathrm{CHO}-\mathrm{CD} 80$ and $\mathrm{CHO}-\mathrm{CD} 86$ but not parental $\mathrm{CHO}$ cells showed significantly increased hexon accumulation after infection with the Ad species B viruses. Similar pattern of hexon staining was seen throughout the slides; captured fields are representative. The elevated levels of hexon accumulation in HeLa, CHO-CD80 and CHOCD86 cells cannot be attributed to the capturing of the original input virus since the number of stained spots within a single cell is much higher than the number of viral particles that could theoretically enter the cell at the MOI of $10 \mathrm{vp} /$ cell used in these experiments.

\section{Discussion}

CD80 and CD86 expressed on the cell surface enhanced the ability of the Ad species B to infect cells. All members of Ad species B exhibited specific binding to the cells expressing CD80 and CD86 followed by E1A mRNA formation after infection of CD80- and CD86-expressing $\mathrm{CHO}$ cells, whereas minimal levels were detected in parental $\mathrm{CHO}$ cells. Therefore, it appears that all members of Ad species B could utilize CD80 and CD86 as cellular attachment receptors. This finding is consistent with our recent report that Ad3 can utilize CD80 and CD86 as cellular attachment receptors (Short et al., 2004), and is compatible with the facts that CD46 serves as a B2-specific receptor (Gaggar et al., 2003) and that Ad3 is using CD46 as a cellular attachment receptor (Sirena et al., 2004). Ad3 utilizes CD80 and CD86 as primary means of cellular entry on human dendritic cells and possibly other hematopoietic cells but on HeLa cells still appears to rely on additional cellular attachment receptors (Short et al., 2004), possibly including CD46. This is likely the case with other members of Ad species B. Ad species B vectors have been shown to efficiently transduce a variety of cells of hematopoietic origin as well as dendritic cells (Knaan-Shanzer et al., 2001; Rea et al., 2001; Sakurai et al., 2003; Segerman et al., 2000; Shayakhmetov et al., 2000; Short et al., 2004); this finding is consistent with the expression of CD80 and CD86 by these cells (Caux et al., 1994; Engel et al., 1994; Lanier et al., 1995). These vectors also infect mature dendritic cells with greater efficiency than they infect immature dendritic cells (Short et al., 2004), which is consistent with the fact that CD80 and CD86 are up-regulated with dendritic cell maturation (Rea et al., 2001). Expression levels of CD80 and CD86 on CHO cells used in this study represent the levels on mature dendritic cells. In fact, in our earlier studies, we characterized physiological expression levels on CD80 and CD86 on human DCs and on these CHO cell lines (Short et al., 2004; Vasu et al., 2003; Holterman et al., unpublished results). Ad species B vectors have also been generally characterized by infecting neoplastic cells with moderate to high efficiencies, especially when compared with other Ad serotypes (Davidoff et al., 1999; Haviv et al., 2002; Shayakhmetov et al., 2002; Von Seggern et al., 2000; Zhang et al., 2003). CD80 and CD86 are dysregulated in several neoplastic contexts resulting in their over-expression (Koyama et al., 1998; Maeda et al., 2000; Mutti et al., 1998).

The finding that Ad species B members can utilize CD80 and CD86 as cellular attachment receptors will help facilitate further development of these vectors for their use in vaccine and 
cancer gene therapy applications. Dendritic cells are potent antigen presenting cells and, therefore, are the target of vaccine strategies (Rea et al., 1999; Rouard et al., 2000; Wan et al., 1997). A complete understanding of the mechanism and consequences of Ad species B infection of these cells may facilitate development of vectors that will stimulate an effective immune response. The in vivo consequences of Ad species B infection via CD80 and CD86 on native dendritic cells have not yet been determined. Additional studies will be needed to determine the suitability of Ad species B vectors for this application.

As more information is gathered about human Ad, it is becoming increasingly clear that most Ad serotypes have evolved a variety of mechanisms for infecting cells of various types. Ad5 uses CAR as its primary cellular receptor (Bergelson et al., 1997; Tomko et al., 2000) with $\alpha_{\mathrm{V}}$ integrins as secondary receptors (Wickham et al., 1993). It was initially proposed that the expression of CAR on a cell was the primary determinant of infection. Although appearing to be the case in most cells in vitro and many in vivo, this scenario does not appear to represent the entire situation. Ad5 can also infect cells by binding to the $\alpha_{2}$ subunit of the MHC class I protein (Hong et al., 1997) and $\alpha 3 \beta 1$ integrins (Salone et al., 2003). Recently, Ad5 infection via heparan sulfate glycosaminoglycans through the shaft domain of the Ad fiber protein has been proposed to play a critical role in infection in vivo in the liver and possibly other organs (Smith et al., 2002). Ad37, a member of species D which does not use CAR, has also been shown to use multiple receptors including CD46 (Wu et al., 2004) and sialic acid residues (Arnberg et al., 2000). A similar scenario of multiple receptors appears to be the case with the Ad species B.

It has already been demonstrated that there are at least three distinct Ad species B cellular attachment receptors. CD80 and CD86 can be utilized by all of the Ad species B, while CD46 appears to be an Ad subspecies B2-specific receptor (Gaggar et al., 2003; Segerman et al., 2003b) which is also utilized by Ad3 (Sirena et al., 2004). However, what role each of these receptors plays in vivo and in the natural context of Ad infection and pathology remains to be determined. This complicates the picture of Ad biology as well as the development of these vectors for therapeutic applications. The situation is not as simple as whether a specific cell type expresses the receptor utilized by the particular serotype, but rather, which combination of receptors the serotype can utilize are expressed by the target cells and what will be the impact of each receptor pathway on the desired cellular effect. Furthermore, the goal of detargeting the native tropism of the virus is no longer achieved by single mutations preventing the interaction of the virus with one receptor but rather, eliminating the native tropism would have to account for all of the receptor-binding sites. Finally, the biological significance of using such a plethora of receptors has yet to be fully understood. For what purpose has Ad evolved the use of such a varied array of cellular proteins as receptors and how does the biological impact on the host vary with the use of the different receptors are questions that remain to be investigated but may be critical in further harnessing these viruses for therapeutic purposes.

\section{Acknowledgements}

We thank Drs. Joanne Douglas, Theresa Strong, Selvarangan Ponnazhagan, and Eric Hunter for their careful review of the manuscript. The work in this manuscript was supported by the National Institutes of Health Grants RO1 CA94084, RO1 CA83821, RO1 CA93796, and P50 CA83591, and the Komen Foundation.

\section{References}

Arnberg N, Edlund K, Kidd AH, Wadell G. Adenovirus type 37 uses sialic acid as a cellular receptor. J Virol 2000;74:42-48. [PubMed: 10590089]

Azuma M, Ito D, Yagita H, Okumura K, Phillips JH, Lanier LL, Somoza C. B70 antigen is a second ligand for CTLA-4 and CD28. Nature 1993;366:76-79. [PubMed: 7694153] 
Bergelson JM, Cunningham JA, Droguett G, Kurt-Jones EA, Krithivas A, Hong JS, Horwitz MS, Crowell RL, Finberg RW. Isolation of a common receptor for Coxsackie B viruses and adenoviruses 2 and 5. Science 1997;275:1320-1323. [PubMed: 9036860]

Caux C, Vanbervliet B, Massacrier C, Azuma M, Okumura K, Lanier LL, Banchereau J. B70/B7-2 is identical to CD86 and is the major functional ligand for CD28 expressed on human dendritic cells. J Exp Med 1994;180:1841-1847. [PubMed: 7525840]

Cohen CJ, Shieh JT, Pickles RJ, Okegawa T, Hsieh JT, Bergelson JM. The coxsackievirus and adenovirus receptor is a transmembrane component of the tight junction. Proc Natl Acad Sci USA 2001;98:1519115196. [PubMed: 11734628]

Davidoff AM, Stevenson SC, McClelland A, Shochat SJ, Vanin EF. Enhanced neuroblastoma transduction for an improved antitumor vaccine. J Surg Res 1999;83:95-99. [PubMed: 10329101]

Engel P, Gribben JG, Freeman GJ, Zhou LJ, Nozawa Y, Abe M, Nadler LM, Wakasa H, Tedder TF. The B7-2 (B70) costimulatory molecule expressed by monocytes and activated B lymphocytes is the CD86 differentiation antigen. Blood 1994;84:1402-1407. [PubMed: 7520767]

Freeman GJ, Freedman AS, Segil JM, Lee G, Whitman JF, Nadler LM. B7, a new member of the Ig superfamily with unique expression on activated and neoplastic B cells. J Immunol 1989;143:27142722. [PubMed: 2794510]

Gaggar A, Shayakhmetov DM, Lieber A. CD46 is a cellular receptor for group B adenoviruses. Nat Med 2003;9:1408-1412. [PubMed: 14566335]

Haviv YS, Blackwell JL, Kanerva A, Nagi P, Krasnykh V, Dmitriev I, Wang M, Naito S, Lei X, Hemminki A, Carey D, Curiel DT. Adenoviral gene therapy for renal cancer requires retargeting to alternative cellular receptors. Cancer Res 2002;62:4273-4281. [PubMed: 12154029]

Hong SS, Karayan L, Tournier J, Curiel DT, Boulanger PA. Adenovirus type 5 fiber knob binds to MHC class I alpha2 domain at the surface of human epithelial and B lymphoblastoid cells. Embo J 1997;16:2294-2306. [PubMed: 9171344]

Kanerva A, Mikheeva GV, Krasnykh V, Coolidge CJ, Lam JT, Mahasreshti PJ, Barker SD, Straughn M, Barnes MN, Alvarez RD, Hemminki A, Curiel DT. Targeting adenovirus to the serotype 3 receptor increases gene transfer efficiency to ovarian cancer cells. Clin Cancer Res 2002;8:275-280. [PubMed: 11801569]

Kim M, Sumerel LA, Belousova N, Lyons GR, Carey DE, Krasnykh V, Douglas JT. The coxsackievirus and adenovirus receptor acts as a tumour suppressor in malignant glioma cells. Br J Cancer 2003;88:1411-1416. [PubMed: 12778071]

Knaan-Shanzer S, Van Der Velde I, Havenga MJ, Lemckert AA, De Vries AA, Valerio D. Highly efficient targeted transduction of undifferentiated human hematopoietic cells by adenoviral vectors displaying fiber knobs of subgroup B. Hum Gene Ther 2001;12:1989-2005. [PubMed: 11686940]

Koyama S, Maruyama T, Adachi S, Nozue M. Expression of costimulatory molecules, B7-1 and B7-2 on human gastric carcinoma. J Cancer Res Clin Oncol 1998;124:383-388. [PubMed: 9719501]

Krasnykh VN, Mikheeva GV, Douglas JT, Curiel DT. Generation of recombinant adenovirus vectors with modified fibers for altering viral tropism. J Virol 1996;70:6839-6846. [PubMed: 8794325]

Lanier LL, O'Fallon S, Somoza C, Phillips JH, Linsley PS, Okumura K, Ito D, Azuma M. CD80 (B7) and CD86 (B70) provide similar costimulatory signals for $\mathrm{T}$ cell proliferation, cytokine production, and generation of CTL. J Immunol 1995;154:97-105. [PubMed: 7527824]

Louis N, Fender P, Barge A, Kitts P, Chroboczek J. Cell-binding domain of adenovirus serotype 2 fiber. J Virol 1994;68:4104-4106. [PubMed: 8189552]

Maeda T, Towatari M, Kosugi H, Saito H. Up-regulation of costimulatory/adhesion molecules by histone deacetylase inhibitors in acute myeloid leukemia cells. Blood 2000;96:3847-3856. [PubMed: 11090069]

Maizel JV Jr, White DO, Scharff MD. The polypeptides of adenovirus. I Evidence for multiple protein components in the virion and a comparison of types 2, 7A, and 12. Virology 1968;36:115-125. [PubMed: 5669982]

Mutti L, Valle MT, Balbi B, Orengo AM, Lazzaro A, Alciato P, Gatti E, Betta PG, Pozzi E. Primary human mesothelioma cells express class II MHC, ICAM-1 and B7-2 and can present recall antigens to autologous blood lymphocytes. Int J Cancer 1998;78:740-749. [PubMed: 9833768] 
Okegawa T, Li Y, Pong RC, Bergelson JM, Zhou J, Hsieh JT. The dual impact of coxsackie and adenovirus receptor expression on human prostate cancer gene therapy. Cancer Res 2000;60:50315036. [PubMed: 11016624]

Rancourt C, Rogers BE, Sosnowski BA, Wang M, Piche A, Pierce GF, Alvarez RD, Siegal GP, Douglas JT, Curiel DT. Basic fibroblast growth factor enhancement of adenovirus-mediated delivery of the herpes simplex virus thymidine kinase gene results in augmented therapeutic benefit in a murine model of ovarian cancer. Clin Cancer Res 1998;4:2455-2461. [PubMed: 9796978]

Rea D, Havenga MJ, van Den Assem M, Sutmuller RP, Lemckert A, Hoeben RC, Bout A, Melief CJ, Offringa R. Highly efficient transduction of human monocyte-derived dendritic cells with subgroup B fiber-modified adenovirus vectors enhances transgene-encoded antigen presentation to cytotoxic T cells. J Immunol 2001;166:5236-5244. [PubMed: 11290808]

Rea D, Schagen FH, Hoeben RC, Mehtali M, Havenga MJ, Toes RE, Melief CJ, Offringa R. Adenoviruses activate human dendritic cells without polarization toward a T-helper type 1-inducing subset. J Virol 1999;73:10245-10253. [PubMed: 10559341]

Roelvink PW, Lizonova A, Lee JG, Li Y, Bergelson JM, Finberg RW, Brough DE, Kovesdi I, Wickham TJ. The coxsackievirus-adenovirus receptor protein can function as a cellular attachment protein for adenovirus serotypes from subgroups A, C, D, E, and F. J Virol 1998;72:7909-7915. [PubMed: 9733828]

Rouard H, Leon A, Klonjkowski B, Marquet J, Tenneze L, Plonquet A, Agrawal SG, Abastado JP, Eloit M, Farcet JP, Delfau-Larue MH. Adenoviral transduction of human 'clinical grade' immature dendritic cells enhances costimulatory molecule expression and T-cell stimulatory capacity. $\mathrm{J}$ Immunol Methods 2000;241:69-81. [PubMed: 10915850]

Russell WC. Update on adenovirus and its vectors. J Gen Virol 2000;81:2573-2604. [PubMed: 11038369]

Sakurai F, Mizuguchi H, Hayakawa T. Efficient gene transfer into human CD34+ cells by an adenovirus type 35 vector. Gene Ther 2003;10:1041-1048. [PubMed: 12776162]

Salone B, Martina Y, Piersanti S, Cundari E, Cherubini G, Franqueville L, Failla CM, Boulanger P, Saggio I. Integrin alpha3beta1 is an alternative cellular receptor for adenovirus serotype 5. J Virol 2003;77:13448-13454. [PubMed: 14645603]

Segerman A, Arnberg N, Erikson A, Lindman K, Wadell G. There are two different species B adenovirus receptors: $\mathrm{SBAR}$, common to species $\mathrm{B} 1$ and $\mathrm{B} 2$ adenoviruses, and $\mathrm{B} 2 \mathrm{AR}$, exclusively used by species B2 adenoviruses. J Virol 2003a;77:1157-1162. [PubMed: 12502832]

Segerman A, Atkinson JP, Marttila M, Dennerquist V, Wadell G, Arnberg N. Adenovirus type 11 uses CD46 as a cellular receptor. J Virol 2003b;77:9183-9191. [PubMed: 12915534]

Segerman A, Mei YF, Wadell G. Adenovirus types 11p and 35p show high binding efficiencies for committed hematopoietic cell lines and are infective to these cell lines. J Virol 2000;74:1457-1467. [PubMed: 10627557]

Shayakhmetov DM, Li ZY, Ni S, Lieber A. Targeting of adenovirus vectors to tumor cells does not enable efficient transduction of breast cancer metastases. Cancer Res 2002;62:1063-1068. [PubMed: 11861383]

Shayakhmetov DM, Papayannopoulou T, Stamatoyannopoulos G, Lieber A. Efficient gene transfer into human CD34(+) cells by a retargeted adenovirus vector. J Virol 2000;74:2567-2583. [PubMed: 10684271]

Short JJ, Pereboev AV, Kawakami Y, Vasu C, Holterman MJ, Curiel DT. Adenovirus serotype 3 utilizes CD80 (B7.1) and CD86 (B7.2) as cellular attachment receptors. Virology 2004;322:349-359. [PubMed: 15110532]

Sirena D, Lilienfeld B, Eisenhut M, Kalin S, Boucke K, Beerli RR, Vogt L, Ruedl C, Bachmann MF, Greber UF, Hemmi S. The human membrane cofactor CD46 is a receptor for species B adenovirus serotype 3. J Virol 2004;78:4454-4462. [PubMed: 15078926]

Smith T, Idamakanti N, Kylefjord H, Rollence M, King L, Kaloss M, Kaleko M, Stevenson SC. In vivo hepatic adenoviral gene delivery occurs independently of the coxsackievirus-adenovirus receptor. Mol Ther 2002;5:770-779. [PubMed: 12027562]

Sterman DH, Treat J, Litzky LA, Amin KM, Coonrod L, Molnar-Kimber K, Recio A, Knox L, Wilson JM, Albelda SM, Kaiser LR. Adenovirus-mediated herpes simplex virus thymidine kinase/ 
ganciclovir gene therapy in patients with localized malignancy: results of a phase I clinical trial in malignant mesothelioma. Hum Gene Ther 1998;9:1083-1092. [PubMed: 9607419]

Stevenson SC, Rollence M, Marshall-Neff J, McClelland A. Selective targeting of human cells by a chimeric adenovirus vector containing a modified fiber protein. J Virol 1997;71:4782-4790. [PubMed: 9151872]

Tomko RP, Johansson CB, Totrov M, Abagyan R, Frisen J, Philipson L. Expression of the adenovirus receptor and its interaction with the fiber knob. Exp Cell Res 2000;255:47-55. [PubMed: 10666333]

Vasu C, Wang A, Gorla SR, Kaithamana S, Prabhakar BS, Holterman MJ. CD80 and CD86 C domains play an important role in receptor binding and co-stimulatory properties. Int Immunol 2003;15:167175. [PubMed: 12578846]

Von Seggern DJ, Huang S, Fleck SK, Stevenson SC, Nemerow GR. Adenovirus vector pseudotyping in fiber-expressing cell lines: improved transduction of Epstein-Barr virus-transformed B cells. J Virol 2000;74:354-362. [PubMed: 10590124]

Walters RW, Freimuth P, Moninger TO, Ganske I, Zabner J, Welsh MJ. Adenovirus fiber disrupts CARmediated intercellular adhesion allowing virus escape. Cell 2002;110:789-799. [PubMed: 12297051]

Wan Y, Bramson J, Carter R, Graham F, Gauldie J. Dendritic cells transduced with an adenoviral vector encoding a model tumor-associated antigen for tumor vaccination. Hum Gene Ther 1997;8:13551363. [PubMed: 9295130]

Wickham TJ, Mathias P, Cheresh DA, Nemerow GR. Integrins alpha v beta 3 and alpha v beta 5 promote adenovirus internalization but not virus attachment. Cell 1993;73:309-319. [PubMed: 8477447]

Wu E, Trauger SA, Pache L, Mullen TM, von Seggern DJ, Siuzdak G, Nemerow GR. Membrane cofactor protein is a receptor for adenoviruses associated with epidemic keratoconjunctivitis. J Virol 2004;78:3897-3905. [PubMed: 15047806]

Zhang LQ, Mei YF, Wadell G. Human adenovirus serotypes 4 and 11 show higher binding affinity and infectivity for endothelial and carcinoma cell lines than serotype 5. J Gen Virol 2003;84:687-695. [PubMed: 12604821]

\section{Abbreviations}

Ad

adenovirus

Ad\#

adenovirus serotype \#

CAR

coxsackie and adenovirus receptor

CHO

Chinese hamster ovary

6-HIS

six consecutive histidine residues

PBSCM

PBS with $\mathrm{Ca}$ and $\mathrm{Mg}$

RGD

Arg-Gly-Asp

TBST

Tris buffered saline with Tween-20

sCD80

sCD86, Recombinant extracellular domains of human CD80 or CD86 fused with Fc region of human IgG1 

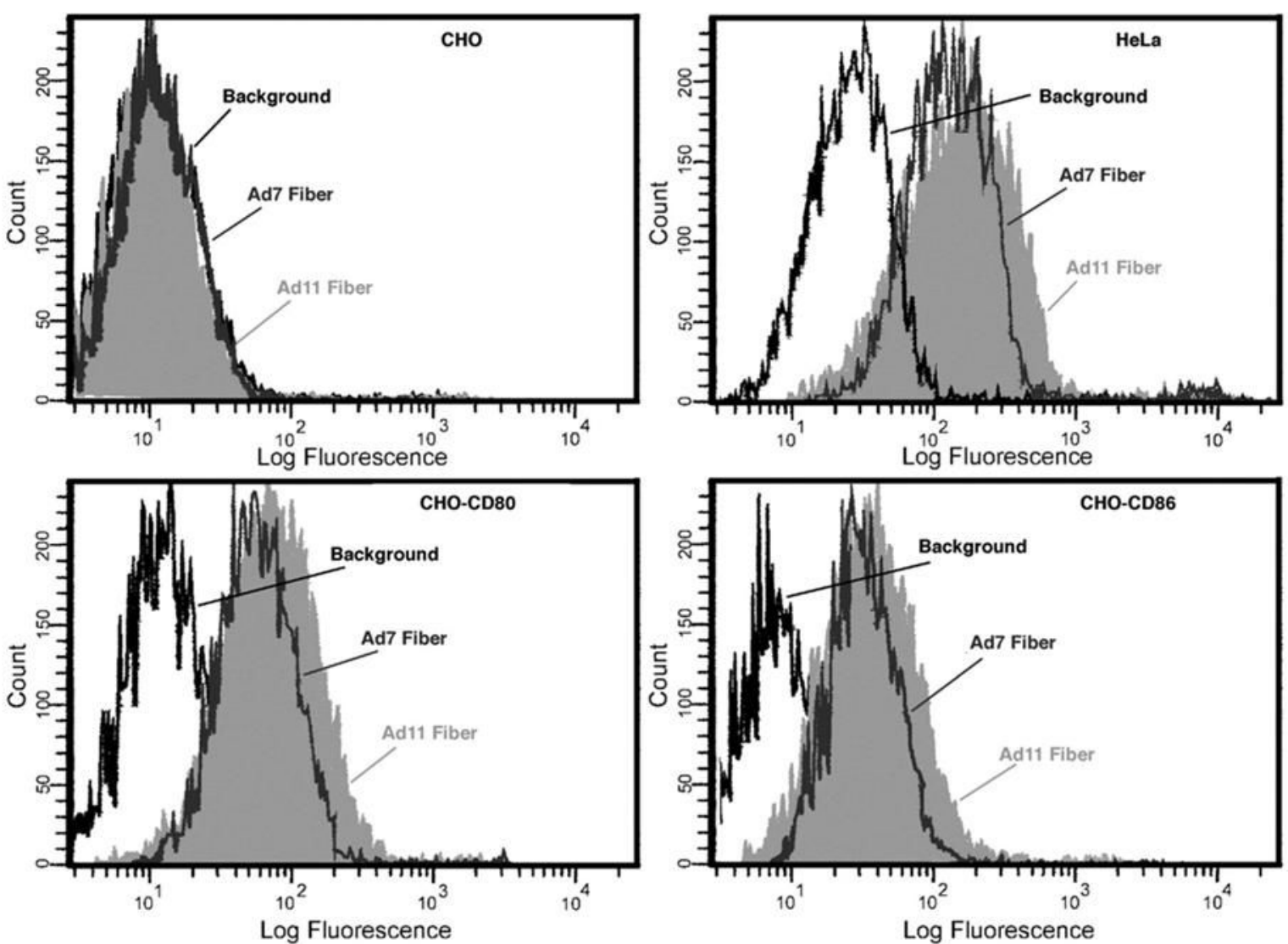

Fig. 1.

Recombinant Ad fiber proteins from Ad subspecies B1 and B2 bind specifically to cells expressing CD80 and CD86. Binding of recombinant Ad7 fiber (gray profile) or Ad11 fiber (shaded profile) to HeLa, CHO, CHO-CD86, and CHO-CD80 cell lines detected via flow cytometry. Cells were incubated with Ad7 fiber, Ad11 fiber, or without Ad fiber (background) followed by anti $6 \mathrm{His} \mathrm{mAb}$ and anti-mouse IgG Abs conjugated to a fluorophore, Alexa 488. Both Ad7 fiber and Ad11 fiber proteins bound specifically to HeLa, CHO-CD80, and CHOCD86 cell lines. Neither Ad7 fiber nor Ad11 fiber proteins bound to CHO cells. 

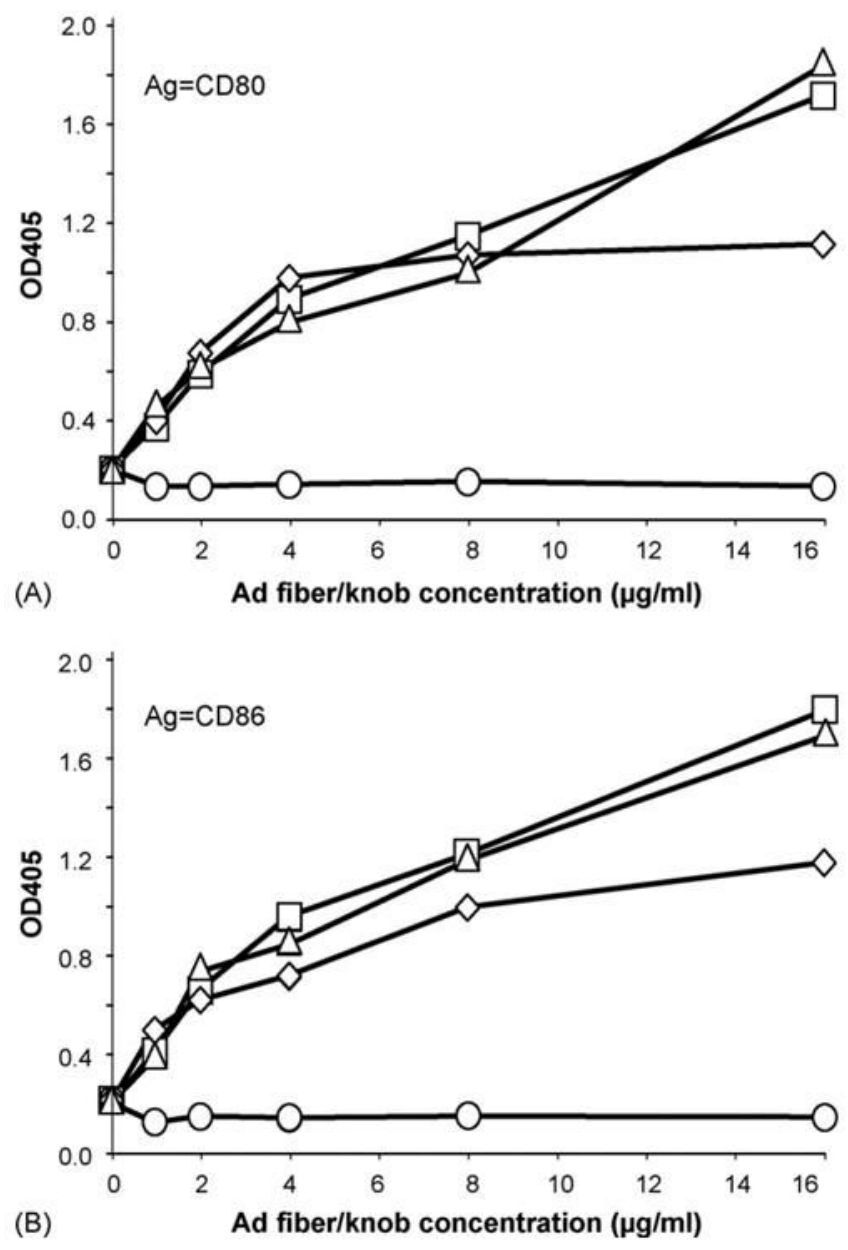

Fig. 2.

Ad fiber proteins from Ad subspecies B1 and B2 interact specifically with CD80 and CD86. ELISA demonstrating the specific interaction between the recombinant Ad3 fiber-knob domain and Ad7 and Ad11 fiber proteins and the soluble recombinant extracellular regions of (A) CD80 and (B) CD86. Indicated amounts of recombinant Ad3 or Ad5 fiber-knob or Ad7 or Ad11 fiber were adsorbed on plastic and incubated with $0.2 \mu \mathrm{g} /$ well of recombinant extracellular domains of CD80 or CD86 fused with Fc region of human IgG1 followed by antihuman IgG Abs conjugated to alkaline phosphatase. Color reaction was developed with $p$ nitrophenyl phosphate. Alkaline phosphatase activity was assayed by measuring the optical density at $405 \mathrm{~nm}$. Ad5 showed no specific binding to CD80 or CD86. Ad3 fiber-knob domain (triangle), Ad5 fiber-knob domain (circle), Ad7 fiber (diamond), Ad11 fiber (square). 

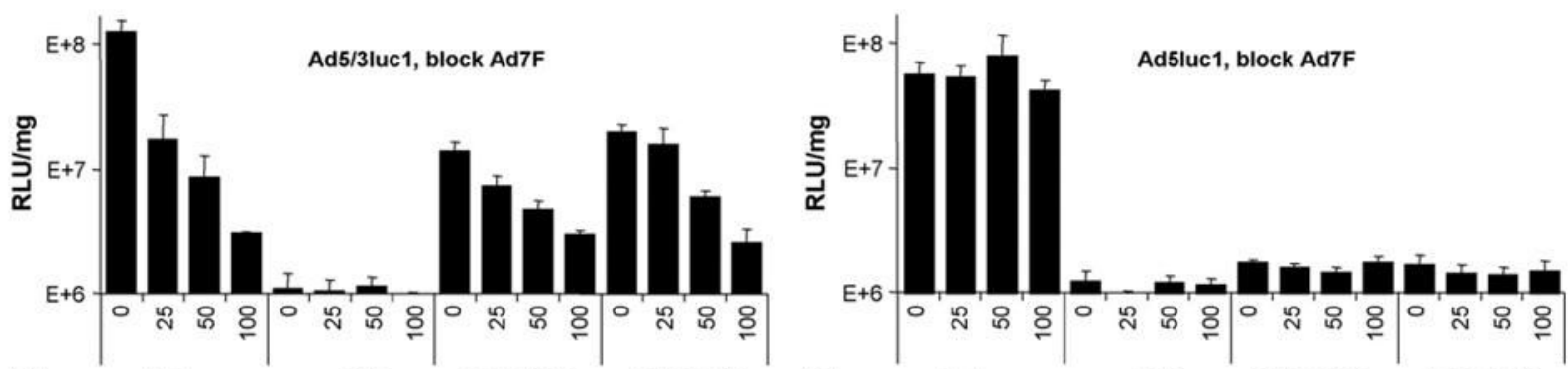

(A)

HeLa

СHо

CHO-CD80

CHO-CD86

(B)

HeLa

CHO

CHO-CD80

CHO-CD86

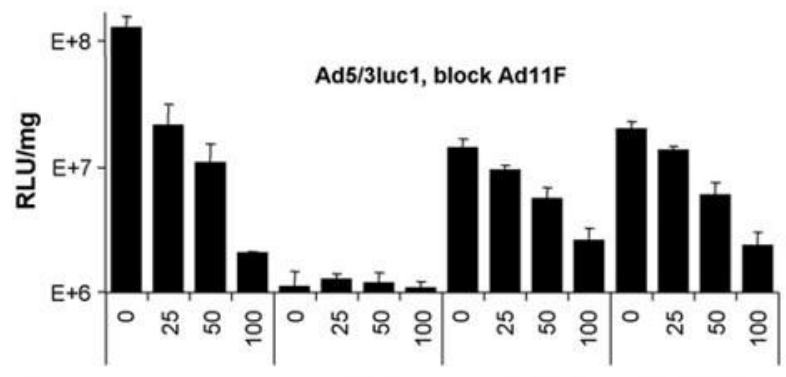

(C)

HeLa

CHO

CHO-CD80

CHO-CD86

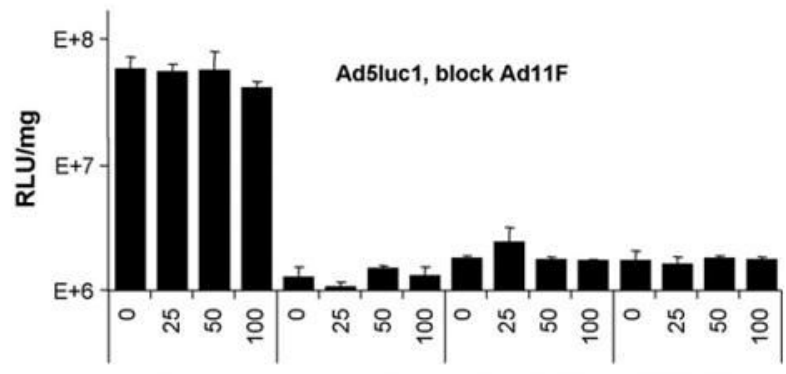

(D)

HeLa

$\mathrm{CHO}$

CHO-CD80

CHO.CD86

Fig. 3.

Recombinant Ad fiber proteins from Ad subspecies B1 and B2 specifically block CD80 and CD86-mediated gene transfer of Ad5/3 pseudotyped vectors. Cells were pre-incubated with indicated amounts of recombinant Ad7 or Ad11 fiber. The cells were then incubated with Ad vectors $(100 \mathrm{vp} / \mathrm{cell})$ or no virus, to determine the background luciferase activity. Virus was removed and the cells were grown in growth media. After $36 \mathrm{~h}$ the cells were lysed and the luciferase assay was performed. Recombinant Ad7 fiber and Ad11 fiber proteins specifically block Ad5/3 pseudotyped vector (Ad5/3luc1) luciferase gene transfer (A and C) but have no effect on Ad5 (Ad5luc1) gene transfer (B and D). Numbers on the horizontal axes are the recombinant fiber concentrations in $\mu \mathrm{g} / \mathrm{ml}$. Relative light units normalized by protein concentration in the sample (RLU/mg). Error bars $= \pm$ S.D. 


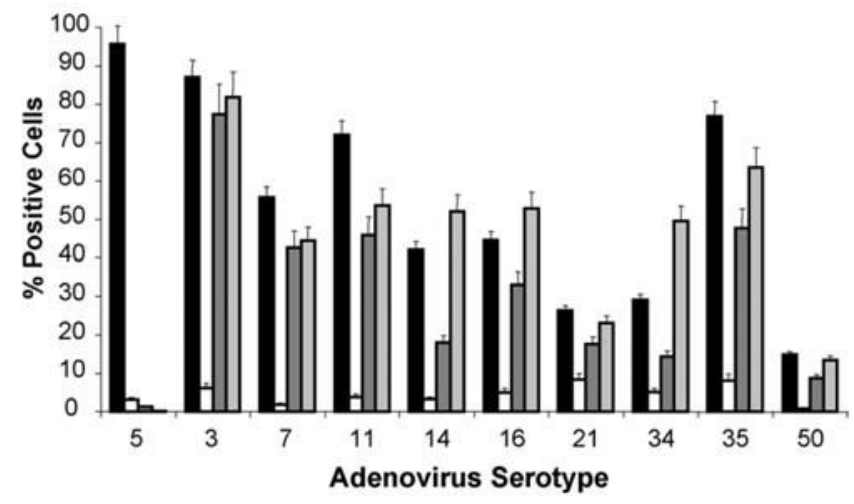

Fig. 4.

Members of Ad species B exhibit specific binding to cells expressing CD80 or CD86. Binding of Ad serotypes with HeLa (black bars), CHO (white bars), CHO-CD80 (dark gray bars), and CHO-CD86 (light gray bars) cells was analyzed. Cells were harvested with Versene and incubated without virus or with 100 vp/cell of Ad3, Ad5, Ad7, Ad11, Ad14, Ad16, Ad21, $\mathrm{Ad} 34, \mathrm{Ad} 35$, or Ad50 for $1.5 \mathrm{~h}$ at $4{ }^{\circ} \mathrm{C}$. Cells were washed and incubated with anti-Ad hexon rabbit antibodies followed by anti-rabbit IgG antibodies conjugated to Alexa 488 and analyzed by flow cytometry. The labeled cells were gated and scored as positive or negative and the percent of positive cells were plotted for each virus. All tested Ad species B members showed increased binding to $\mathrm{CHO}-\mathrm{CD} 80$ and $\mathrm{CHO}-\mathrm{CD} 86$ vs. $\mathrm{CHO}$ cells. Ad5 did not show any increased binding to $\mathrm{CHO}-\mathrm{CD} 80$ or $\mathrm{CHO}-\mathrm{CD} 86$ vs. $\mathrm{CHO}$ cells. Error bars $= \pm$ S.D. 


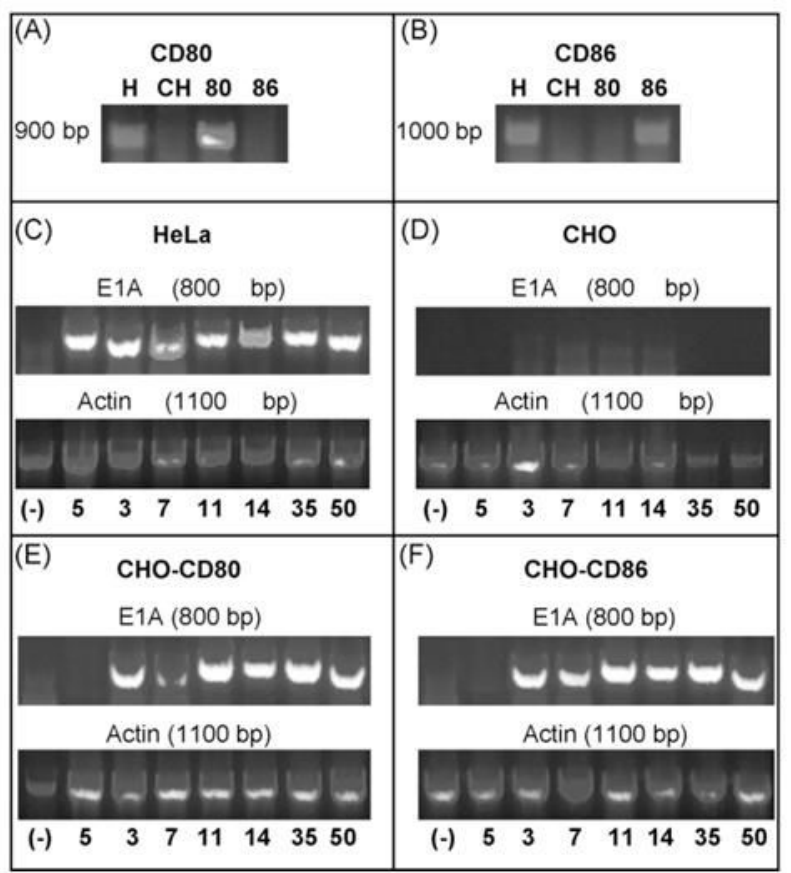

Fig. 5.

Surface expression of CD80 and CD86 allows infection by members of the Ad species B. RTPCR demonstrating RNA transcripts of (A) CD80 in HeLa (H) and CHO-CD80 (80) cells and (B) CD86 in HeLa and CHO-CD86 cells (86), but neither in parental CHO cells (CH). E1 RNA transcripts after infection with Ad species B serotypes are present for the Ad species B serotypes in (C) HeLa, (E) CHO-CD80, and (F) CHO-CD86 cells but not (D) CHO cells. Numbers below lanes represent Ad serotype used. (-) is a no template negative control. 
Fig. 6.
$\mathrm{CHO}$
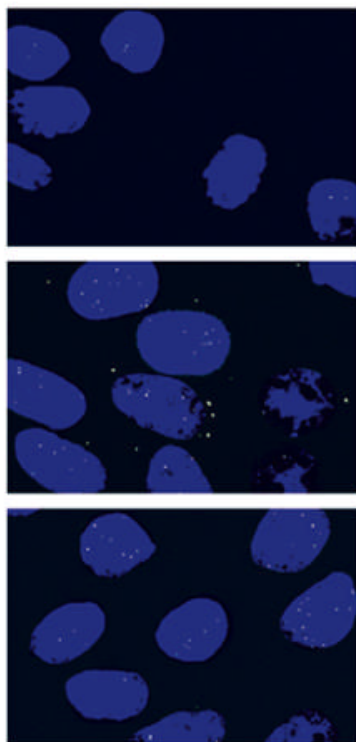

HeLa
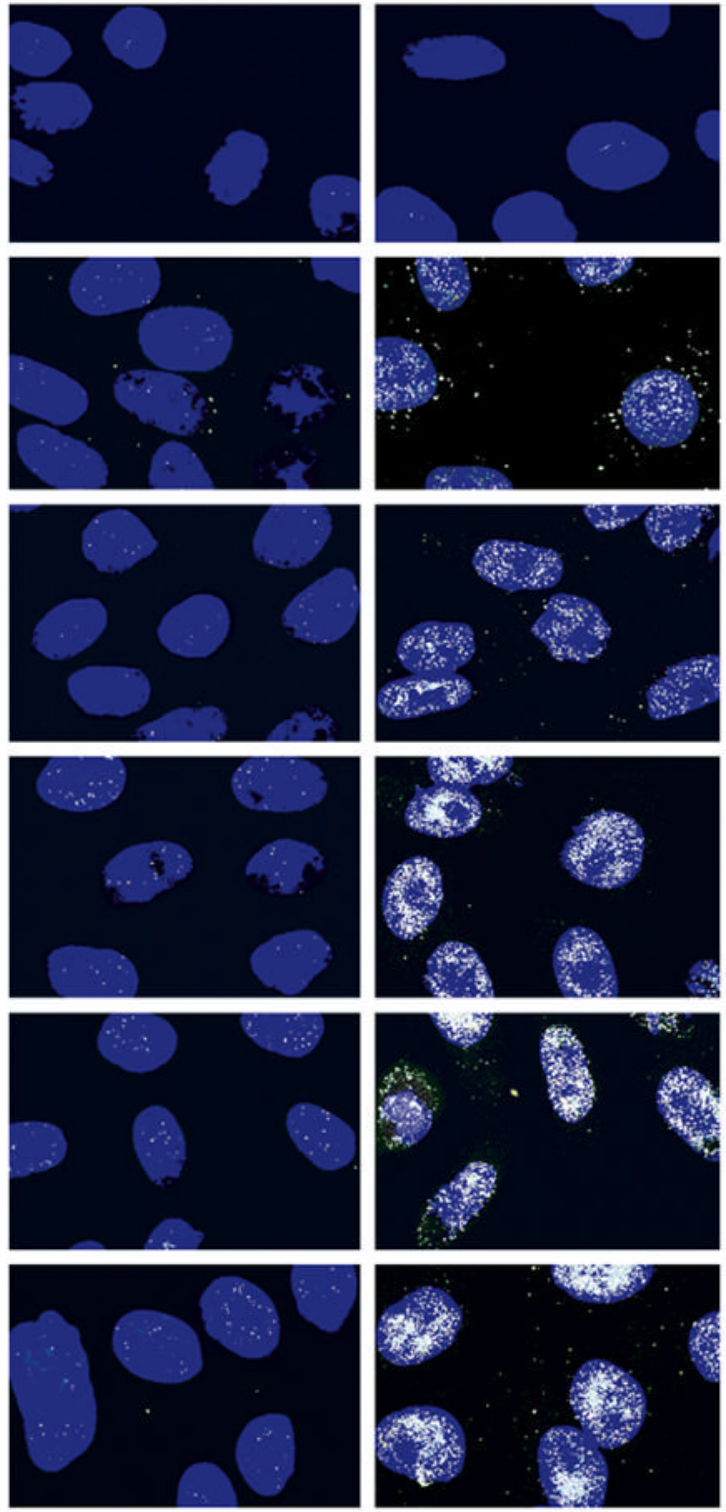
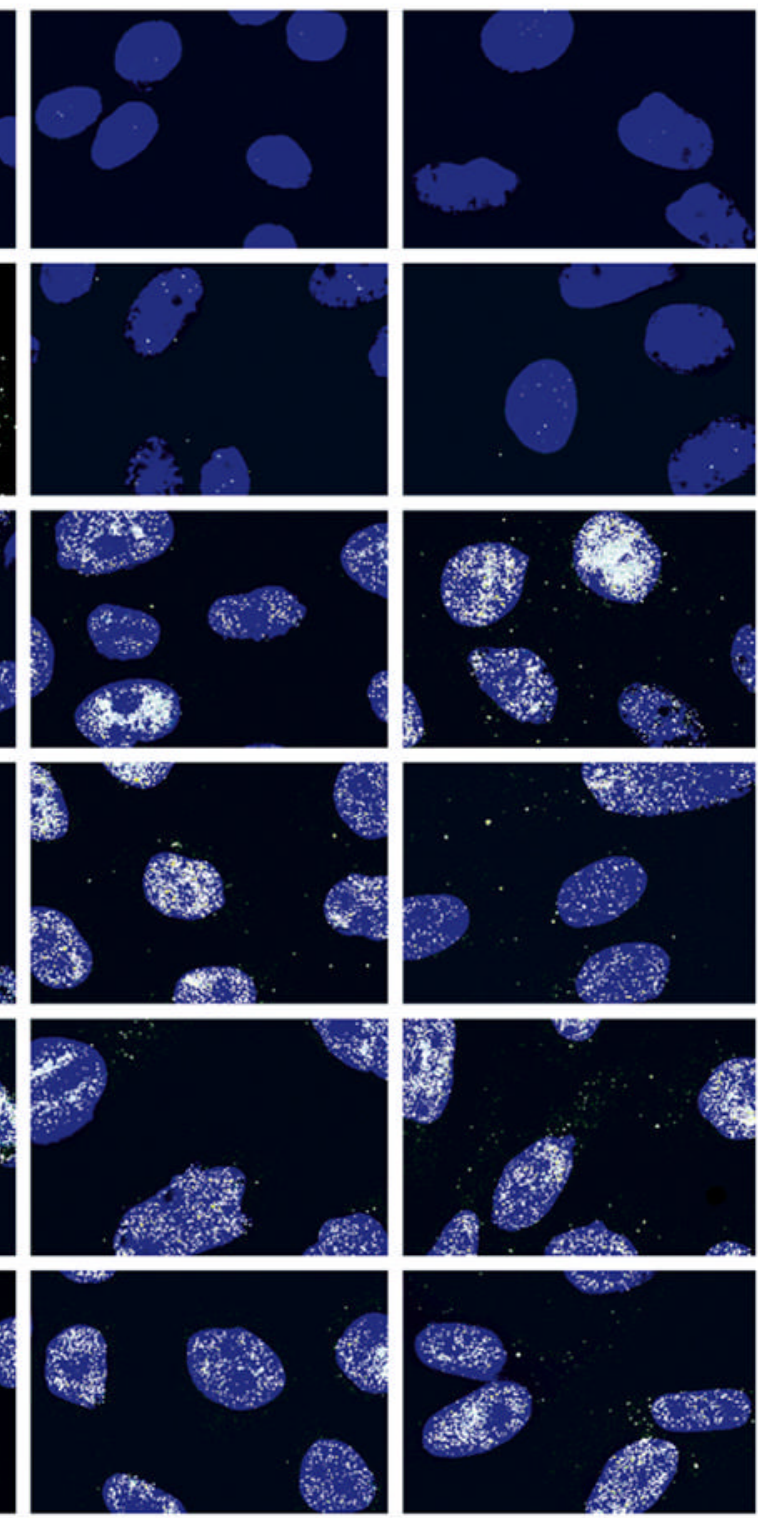

Hexon accumulation of Ad species B members from cells expressing CD80 and CD86. HeLa cells and parental CHO cells without expression of CD80 or CD86 and CHO derivative cell lines expressing CD80 or CD86 were infected with wild-type Ad subspecies B1 (Ad3, Ad7) and B2 (Ad11, Ad35) and Ad5. Expression of the Ad capsid protein hexon (white) was detected $36 \mathrm{~h}$ post-infection by immunofluorescence. $\mathrm{CHO}-\mathrm{CD} 80$ and $\mathrm{CHO}-\mathrm{CD} 86$ cells were efficiently infected by only the Ad species B, while the HeLa cells were infected by all of the Ad serotypes and the CHO cells were only minimally infected by all of the Ad serotypes. Cell nuclei (blue) stained with Hoechst 33342. (-) is for uninfected cells. Similar pattern of hexon staining was seen throughout the slides; captured fields are representative. 